\title{
Quantidade de Indivíduos que Utilizaram Computador no Brasil entre 2008 e 2016
}

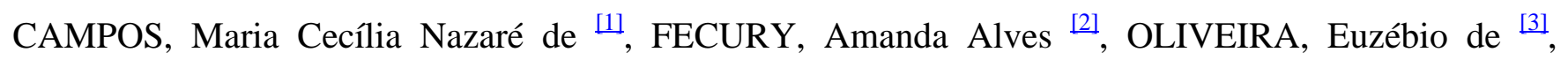
DENDASCK, Carla Viana ${ }^{[4]}$, DIAS, Cláudio Alberto Gellis de Mattos [5]

CAMPOS, Maria Cecília Nazaré de, et.al. Quantidade de Indivíduos que Utilizaram Computador no Brasil entre 2008 e 2016. Revista Científica Multidisciplinar Núcleo do Conhecimento. Edição 09. Ano 02, Vol. 04. pp 125-132, Dezembro de 2017. ISSN:2448-0959

\section{RESUMO}

Um computador é essencialmente têm a função de processar, armazenar e controlar a entrada e saída de dados, o computador se tornou uma ferramenta importante em vários âmbitos da sociedade, logo, os indivíduos que não tiveram oportunidades para acompanhar esse desenvolvimento parecem ter sido excluídos, dando início a mais uma desigualdade social, a exclusão digital. A pesquisa foi realizada no banco de dados CETIC (http://data.cetic.br/cetic/). Embora a velocidade do uso do computador aumente e se torne cada vez mais presente no cotidiano dos indivíduos, ainda há fatores como a diferença da distribuição de renda para as regiões do Brasil, o que parece restringir o acesso a um grupo específico, potencializando a desigualdade digital

Palavras-chave: Computador, Utilização, Desigualdade Digital.

\section{INTRODUÇÃO}

Um computador é essencialmente formado por uma memória e um processador que interligados a outros componentes têm a função de processar, armazenar e controlar a entrada e saída de dados (WOILER, 1970). Esses dados são interpretados e convertidos em diversos tipos de informações (BECKER, 2015) que, por meio de uma linguagem computacional, se modificam em textos, imagens, vídeos e outros conteúdos (FILHO, 2017). Tais informações geram um conhecimento cada vez mais amplo, uma vez que, um computador conectado à internet, possibilita o aumento da velocidade de comunicação interpessoal e também entre instituições (MARTINS e SILVA, 2013).

Nos últimos anos, o computador se tornou uma ferramenta importante em vários âmbitos da sociedade, 
logo, os indivíduos que não tiveram oportunidades para acompanhar esse desenvolvimento parecem ter sido excluídos (CRUZ-CUNHA et al., 2014), dando início a mais uma desigualdade social, a exclusão digital. Tal exclusão impede a participação de pessoas desprovidas de recurso e conhecimento sobre o mundo tecnológico. Atualmente, as tentativas de inclusão digital parecem ser insuficientes para reduzir o índice de desigualdade, pois possivelmente se os projetos que promovem essa inclusão fossem consolidados em políticas públicas, talvez aumentassem o acesso de mais pessoas às novas tecnologias de informação (GROSSI et al., 2013.

\section{OBJETIVOS}

Mostrar a quantidade de indivíduos que utilizaram computador no Brasil entre 2008 e 2016.

\section{MÉTODO}

Pesquisa realizada no banco de dados CETIC (http://data.cetic.br/cetic/). Acessando a aba "explore", foi selecionada a opção "usuários" e a opção "tabela". Clicou-se no filtro "Indicador" a opção "alterar". Selecionou-se a alternativa "B1 - INDIVÍDUOS QUE JÁ UTILIZARAM UM COMPUTADOR” e, em seguida, foram selecionadas no filtro as categorias "Sim" e "Não" para todos os tipos de Linhas posteriormente selecionadas. Para cada opção foram coletados os dados de todos os anos entre o período de 2008 a 2016 na área "Ano". As linhas utilizadas foram: "Total", "Área"," Classe social", "Faixa etária" e "Renda familiar". A pesquisa bibliográfica foi realizada em artigos científicos, em computadores do Instituto Federal de Educação, Ciência e Tecnologia do Amapá, Campus Macapá, situado à Rodovia BR 210 KM 3, s/n - Bairro Brasil Novo. CEP: 68.909-398, Macapá, Amapá, Brasil. Os dados foram compilados no aplicativo Excel, componente do pacote Office da Microsoft Corporation.

\section{RESULTADOS}

A figura 1 mostra a média da porcentagem de indivíduos que já utilizaram um computador no Brasil entre os anos de 2008 a 2016. Os resultados mostram que a maioria das pessoas já utilizou um computador. 


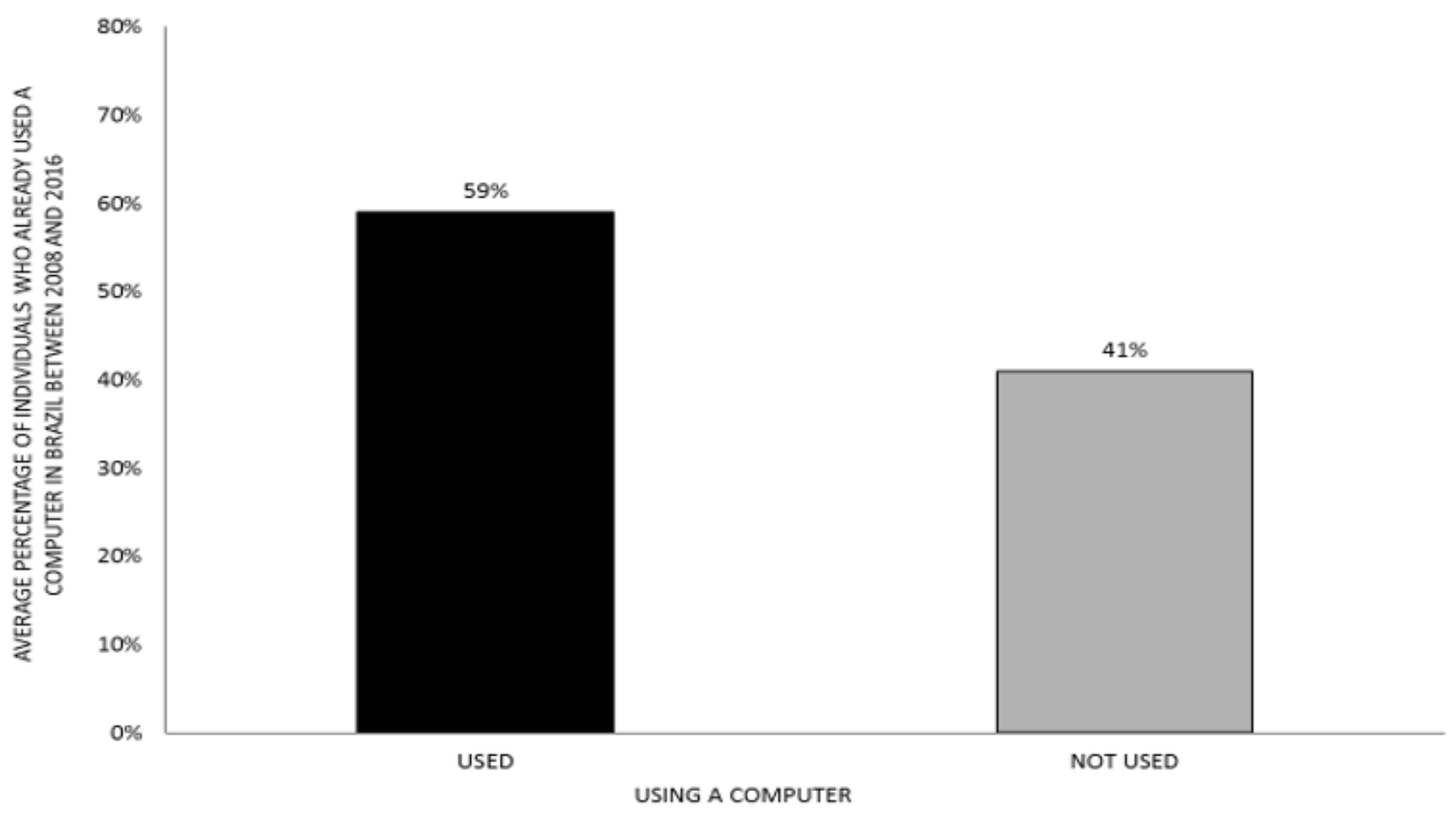

Figure 1 - Percentagem média de indivíduos que usaram um computador no Brasil entre 2008 e 2016.

A figura 2 mostra a média da porcentagem de indivíduos que já utilizaram um computador no Brasil, por ano. A tendência de uso aumentou ao longo dos anos e, consequentemente, a tendência de não utilização diminuiu. 


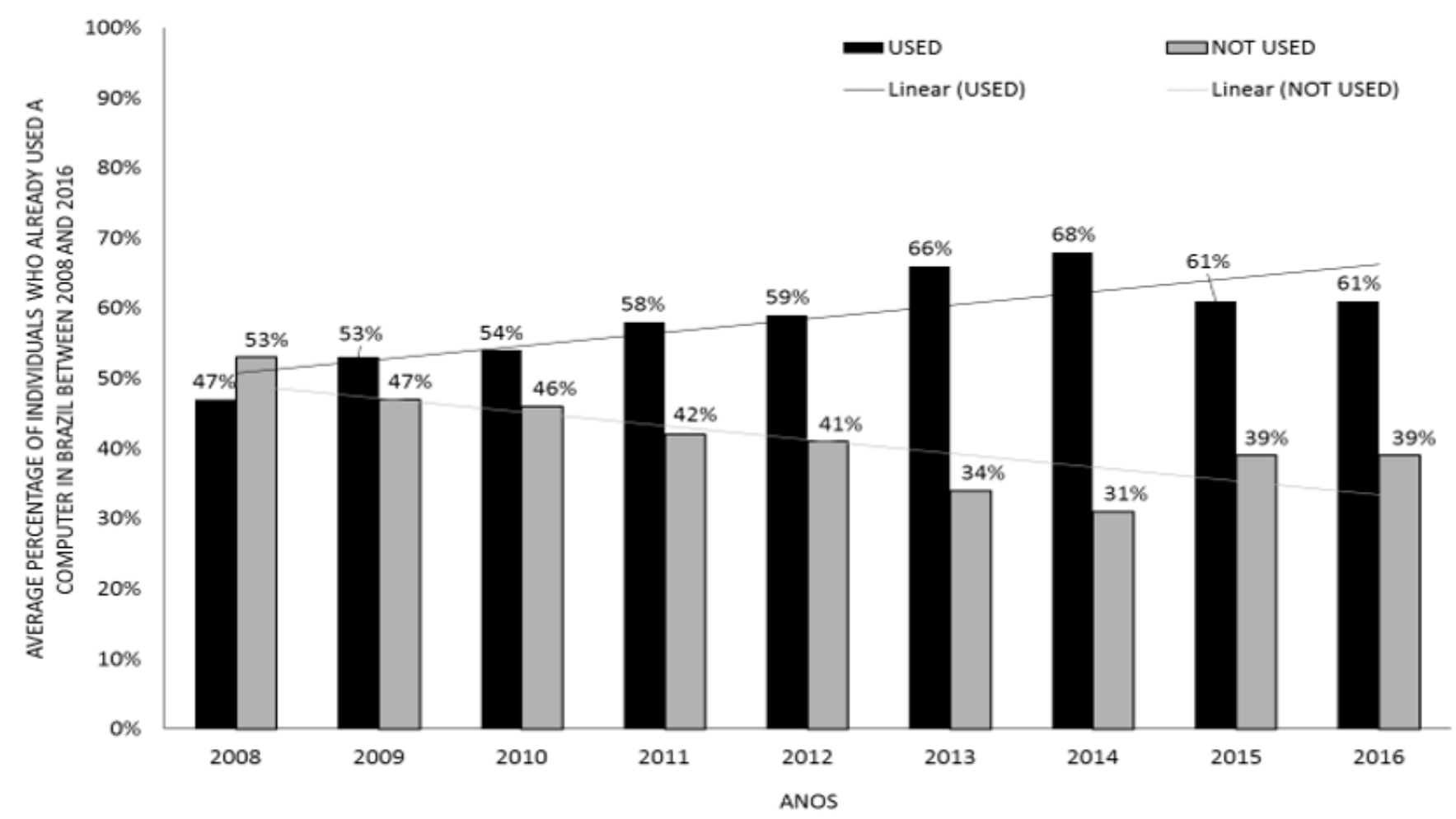

Figure 2 - Percentagem média de indivíduos que usaram um computador no Brasil entre 2008 e 2016.

A figura 3 mostra a média da porcentagem de indivíduos que já utilizaram um computador no Brasil, por faixa etária. O maior número de usuários se concentra na faixa etária até 34 anos. 


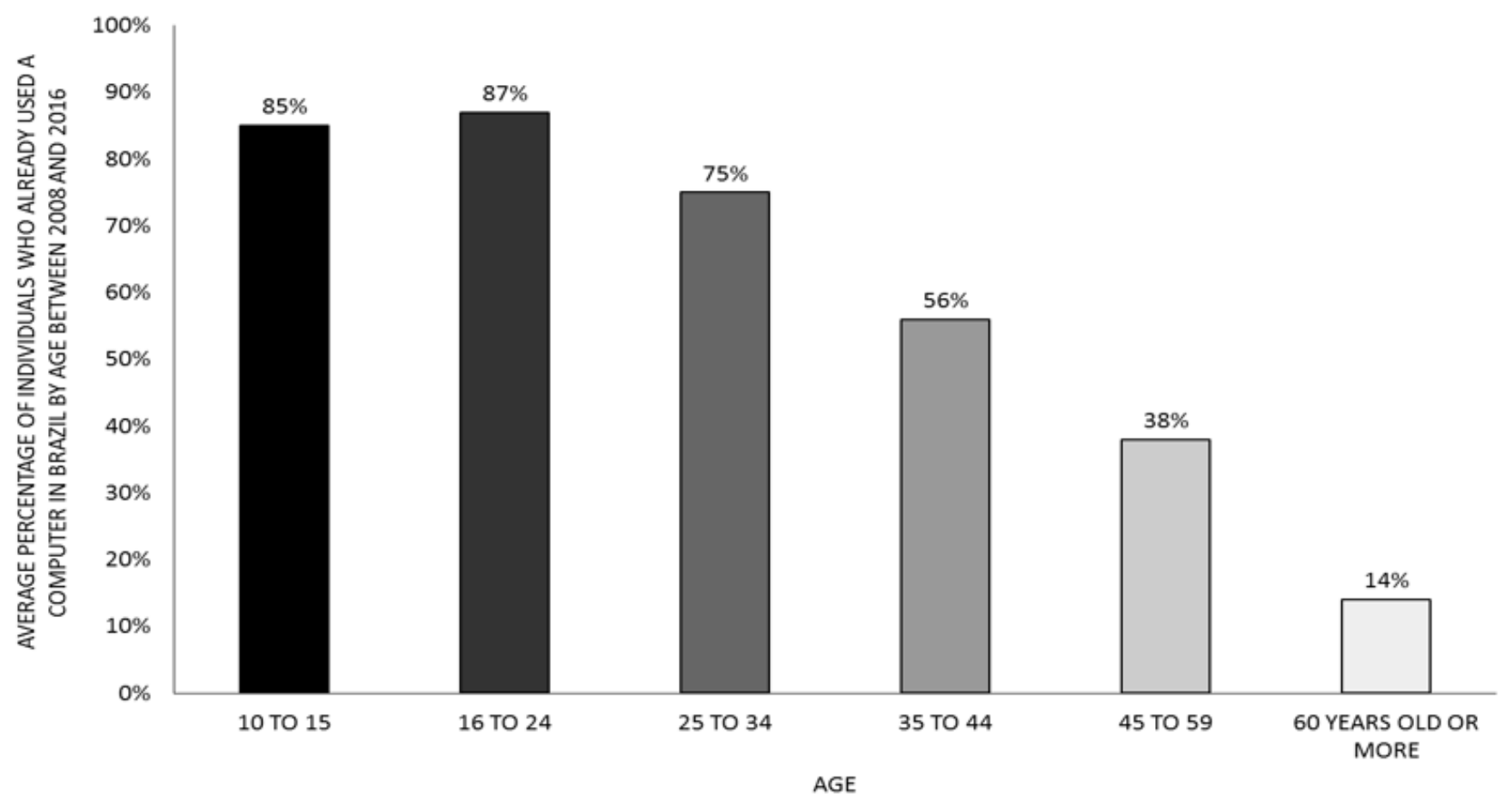

Figura 3 - Percentagem média de indivíduos que já utilizaram um computador no Brasil por faixa etária entre 2008 e 2016.

A figura 4 mostra a média da porcentagem de indivíduos que já utilizaram um computador no Brasil entre 2008 e 2016 divididos por faixa etária. A tendência de uso entre pessoas de 35 a 59 anos parece aumentar com maior velocidade em comparação ao de pessoas mais jovens. 


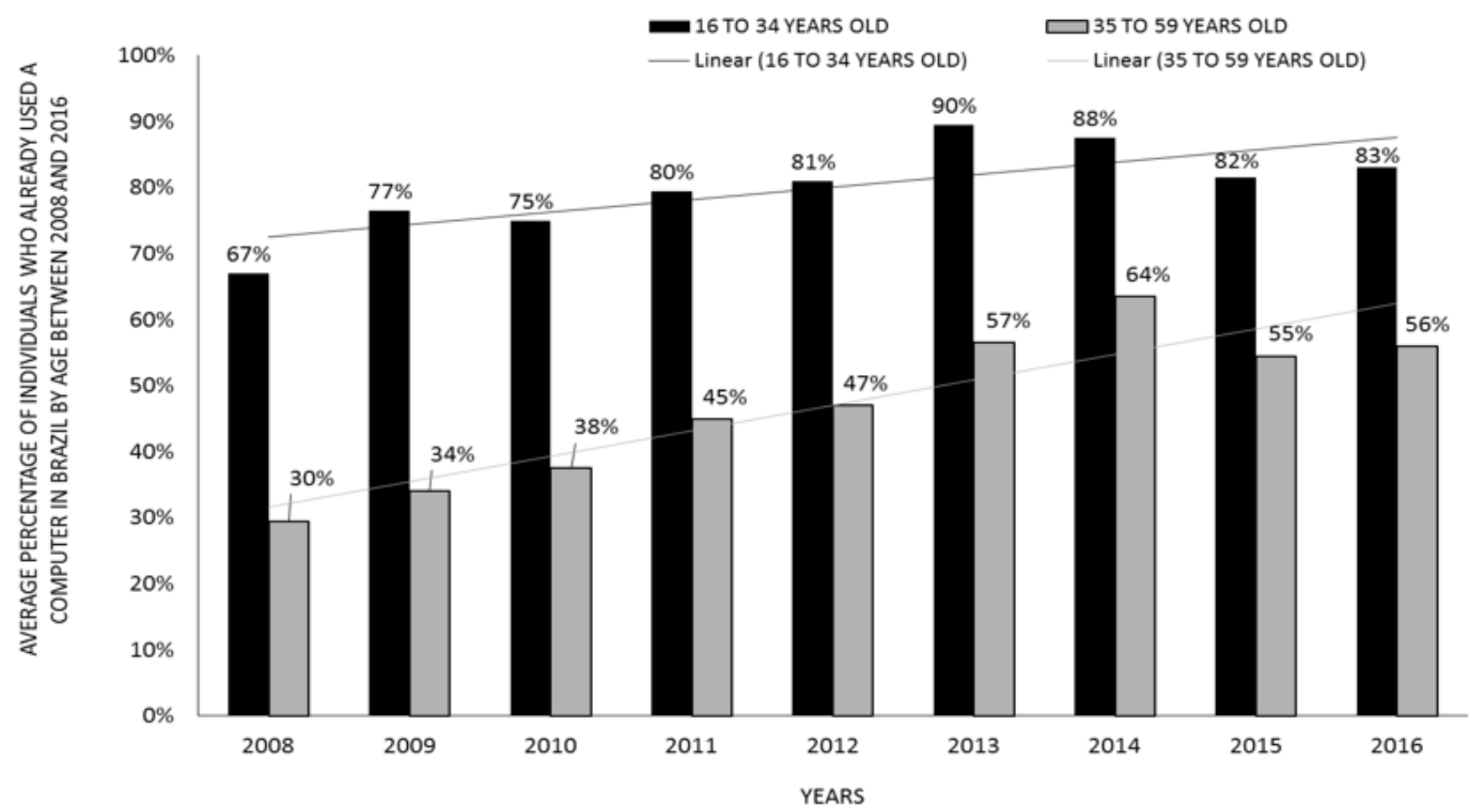

Figura 4 - Percentagem média de indivíduos que já utilizaram um computador no Brasil por faixa etária entre 2008 e 2016.

A figura 5 mostra a média da porcentagem de indivíduos que já utilizaram um computador no Brasil divididos por região. O uso é maior na região Sudeste, seguida pelas regiões Centro-oeste e Sul. As regiões Norte e Nordeste tem, respectivamente, as menores medias de utilização. 


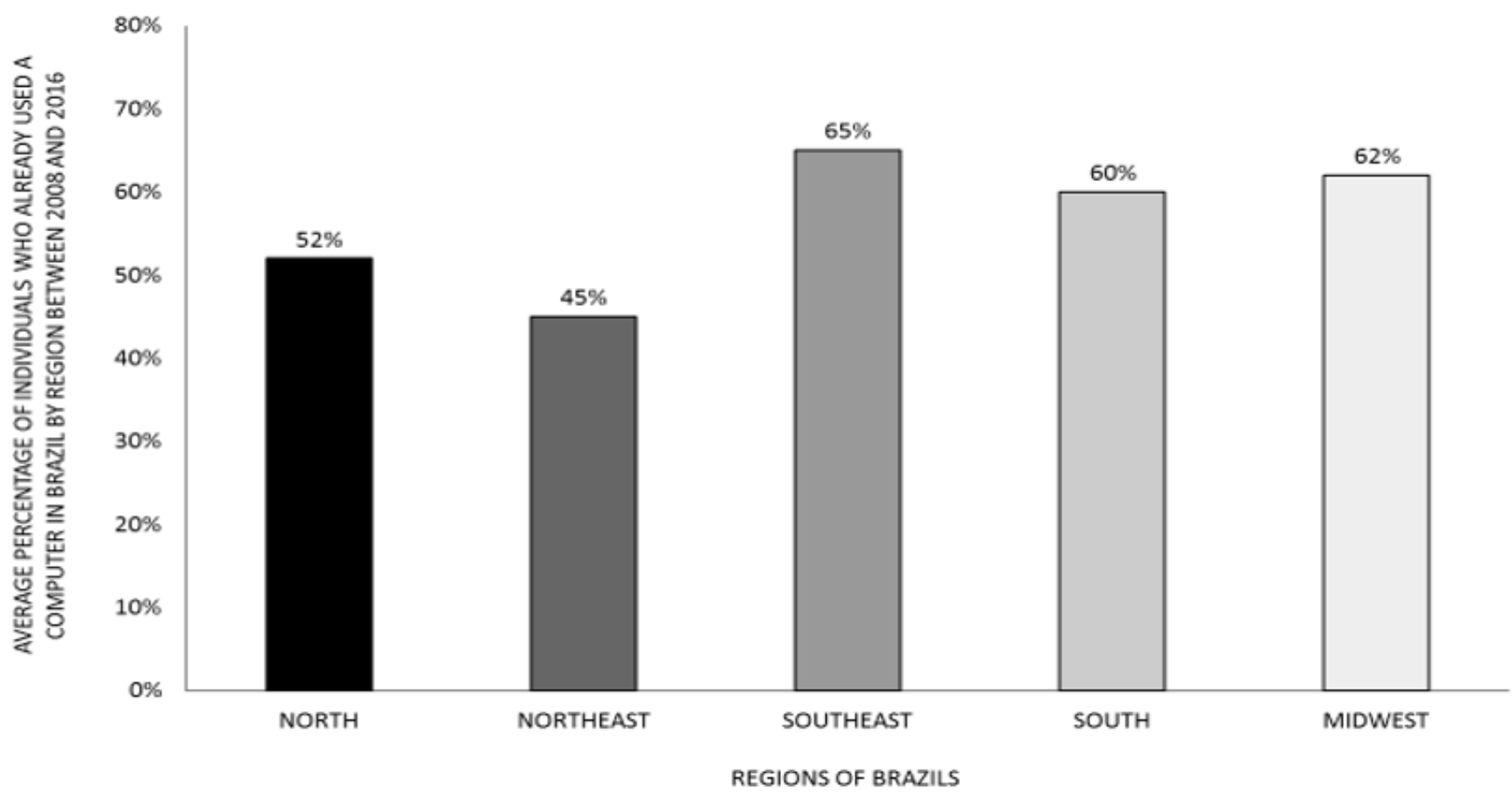

Figure 5 - Percentagem média de indivíduos que usaram um computador no Brasil por região entre 2008 e 2016.

A figura 6 mostra a média da porcentagem de indivíduos que já utilizaram um computador no Brasil divididos por área. Os dados mostram que existem mais usuários na área urbana do que na área rural. 


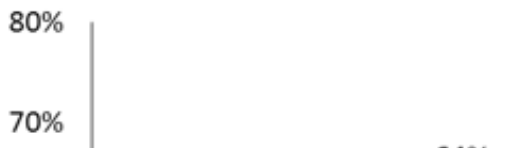

$60 \%$

$50 \%$

$50 \%$

$40 \%$

$30 \%$

$20 \%$

$10 \%$

$0 \%$

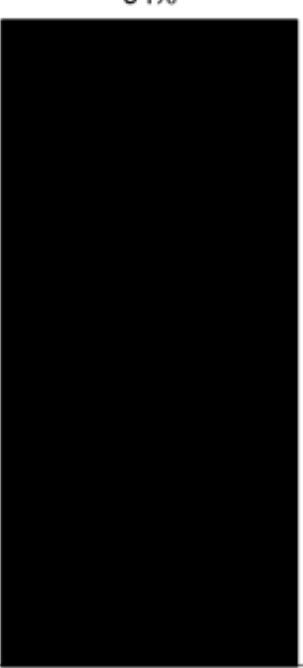

URBAN
$32 \%$

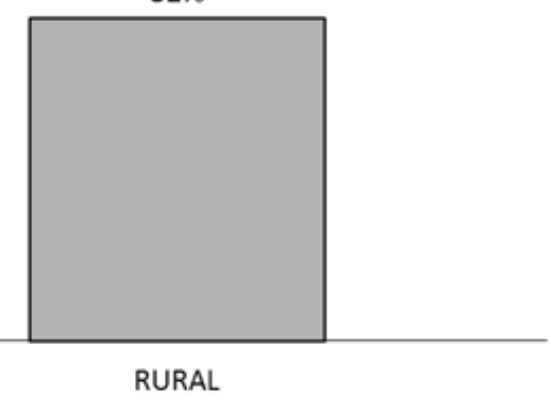

AREA

Figure 6 - Percentagem média de indivíduos que usaram um computador no Brasil por área entre 2008 e 2016.

A figura 7 mostra a média da porcentagem de indivíduos que já utilizaram um computador no Brasil, por classe. Há mais usuários pertencentes às classes $\mathrm{A}$ e $\mathrm{B}$ em comparação às classes $\mathrm{C}$ e DE. 


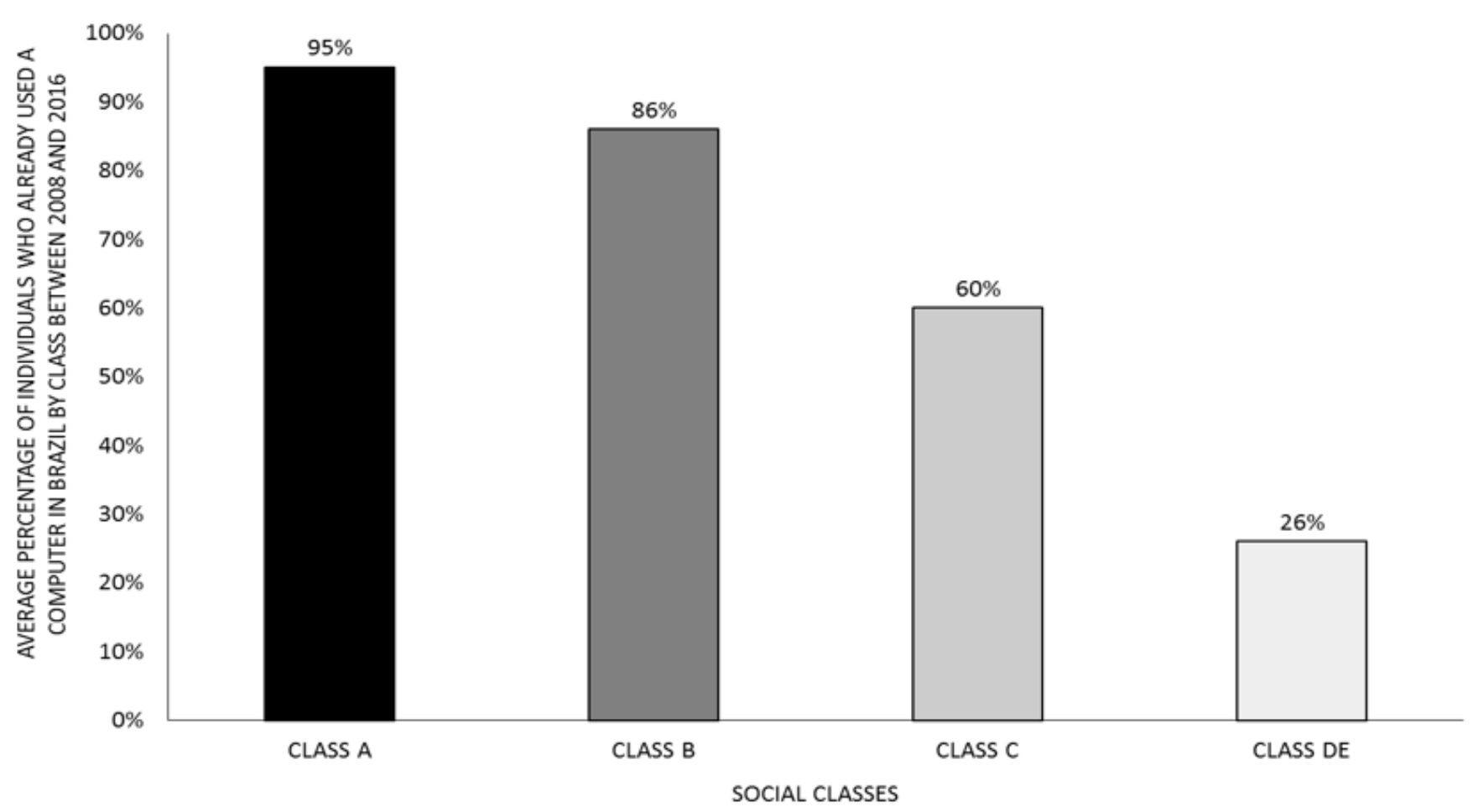

Figura 7 - Percentagem média de indivíduos que já usaram um computador no Brasil por classe entre 2008 e 2016.

\section{DISCUSSÃO}

O computador se difundiu na sociedade brasileira nos últimos anos, se tornando cada vez mais utilizado (Figura 1) e necessário por sua rapidez e precisão que parece ter facilitado e inovado o modo como os indivíduos trabalham e se relacionam. O aumento da utilização dessa tecnologia (Figura 2) fez com que se tornasse uma importante ferramenta para a comunicação e aquisição de informações em diversas áreas (DUDZIAK, 2010).

O uso do computador parece ser maior por pessoas mais jovens (figura 3) que nasceram na era digital, os chamados nativos digitais. A tecnologia normalmente é inserida de maneira natural na sua formação social. Em contrapartida os imigrantes digitais, representados pela população mais velha, tiveram que se adaptar ao desenvolvimento da tecnologia (PASSARELLI et al., 2014). Esse processo de adaptação e aprendizagem é gradativo e ainda não se totalizou, por isso os imigrantes digitais ainda têm menor acesso e consequentemente encontram dificuldades para serem incluídos digitalmente na sociedade (PETRELLA et al., 2016).

Embora o processo de adaptação seja longo, o número de usuários idosos parece ter aumentado (figura 4) e conforme o interesse em aprender e se incluir digitalmente cresce, a tendência é que daqui a alguns anos todos tenham acesso ao uso do computador independente da faixa etária (SALES et al., 2014).

A partir da diferença na utilização de computadores entre as regiões do Brasil (figura 5) percebe-se a dependência na relação dos fatores socioeconômicos com a inclusão digital. As regiões menos desenvolvidas como Norte e Nordeste têm menor acesso em relação às regiões Sudeste, Centro-oeste e 
Sul (PASSARELLI et al., 2014). Isso parece se dar pela diferença na velocidade do processo de urbanização de cada região, fator diretamente ligado à economia e desenvolvimento do país (CAMARANO e CARNEIRO,2016).

As características sociais das zonas urbana e rural são bastante distintas devido ao processo de industrialização da primeira, isso parece se tornar mais evidente quando as ofertas de infraestrutura para o acesso ao uso do computador são maiores na área urbana (figura 6). Essa zona parece receber maior benefício em comparação à zona rural, uma vez que possui maior concentração de renda. Tal disparidade pode acarretar consequências em relação à posse de informações por meio da tecnologia, resultando na limitação de oportunidade de conhecimento e comunicação dos habitantes da zona rural (PASSARELLI et al., 2014).

As classes A e B parecem ter mais oportunidade de acesso do que as C e DE (figura 7), uma vez que, a desigualdade na distribuição de renda no Brasil, reflexo de uma possível má administração governamental, parece incidir também na restrição à educação e informação digital por pessoas pertencentes às classes menos favorecidas (GROSSI et al., 2013).

\section{CONCLUSÃO}

Tendo em vista os aspectos observados percebe-se que, embora a velocidade do uso do computador aumente e se torne cada vez mais presente no cotidiano dos indivíduos, ainda há fatores como a diferença da distribuição de renda para as regiões do Brasil, o que parece restringir o acesso a um grupo específico, potencializando a desigualdade digital. Há projetos sociais que tentam promover a inclusão digital, porém, são insuficientes para diminuir consideravelmente essa desigualdade até que se tornem políticas públicas que de fato possibilitem oportunidades àqueles menos beneficiados.

\section{REFERENCIAS}

BECKER J. L. Estatística Básica: Transformando Dados em informação. Bookman. Porto Alegre, 2015.

CAMARANO A. A. e CARNEIRO I. G. Padrões de Formação de Família por Regiões Brasileiras e Grupos Sociais: Diferenças ou semelhanças?. XI Encontro Nacional de Estudos Populacionais da ABEP. 2016

CRUZ-CUNHA M. M., SIMÕES R., VARAJÃO J., MIRANDA I. O impacto da exclusão digital na utilização potencial de um mercado eletrônico de serviços de cuidados de saúde e serviços sociais. RISTI - Revista Ibérica de Sistemas e Tecnologias de informação. Porto, 2014.

DUDZIAK E. A. Competência informacional e midiática no ensino superior: Desafios e propostas para o Brasil. Prisma.com. São Paulo, 2010.

FILHO R. G. N. O conceito de processamento de dados. <www.di.ufpb.br/raimundo/Generalidades/ProcDados.html>. Acesso em: dez, 2017.

GROSSI M. G. R., COSTA J. W., SANTOS A. J. A exclusão digital: o reflexo da desigualdade social no 
Brasil. Nuances: estudos sobre Educação. V 24. São Paulo, 2013.

MARTINS B. S., SILVA F. D. S. O uso do computador e da internet como catalisadores do conhecimento. Universidade Estadual de Goiás, 2013.

PASSARELI B., JUNQUEIRA A. H., ANGELUCI A. C. B. Os nativos digitais no Brasil e seus comportamentos diante das telas. Redalyc. V 8. São Paulo, 2014.

PETRElla S., PINTO M., PEREIRA S. a. O Idoso e a Educação para os Media: Novos Desafios entre Envelhecimento e Exclusão Social. $8^{\circ}$ SOPCOM: "Comunicação Global, Cultura e Tecnologia". Pg 150-155. 2016

SAlES M. B., AMARAL M. A., JUNIOR I. G. S., SALES A. B. Tecnologias de Informação e Comunicação via Web: Preferências de uso de um grupo de usuários idosos. Kairós: Gerontologia. Santa Catarina, 2014.

$11]$ Técnica em Redes de Computadores pelo Instituto Federal do Amapá - IFAP

[2] Biomédica. Doutora em Doenças Tropicais. Docente e Pesquisadora da Universidade Federal do Amapá, AP. Pesquisador colaboradora do Núcleo de Medicina Tropical da UFPA (NMT-UFPA).

${ }^{[3]}$ Biólogo. Doutor em Medicina/Doenças Tropicais. Docente e Pesquisador na Universidade Federal do Pará - UFPA

${ }^{[4]}$ Doutora em Psicanálise Clínica, Pesquisadora pelo Centro de Pesquisa e Estudos Avançados

${ }^{[5]}$ Biólogo. Doutor em Teoria e Pesquisa do Comportamento. Docente e Pesquisador do Instituto Federal do Amapá - IFAP

\section{PUBLIQUE SEU ARTIGO CIENTÍFICO EM:}

https://www.nucleodoconhecimento.com.br/enviar-artigo-cientifico-para-submissao 